\title{
Sequential Majoritarian Blotto Games
}

Max Planck Institute for Tax Law and Public Finance

Working Paper 2018 - 05

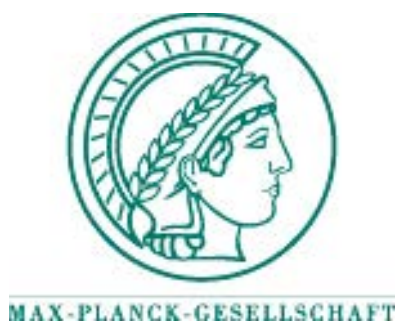

Max Planck Institute for

Tax Law and Public Finance

Department of Business and Tax Law

Department of Public Economics

http:/ / www.tax.mpg.de 
Working papers of the Max Planck Institute for Tax Law and Public Finance Research Paper Series serve to disseminate the research results of work in progress prior to publication to encourage the exchange of ideas and academic debate. Inclusion of a paper in the Research Paper Series does not constitute publication and should not limit publication in any other venue. The preprints published by the Max Planck Institute for Tax Law and Public Finance represent the views of the respective author(s) and not of the Institute as a whole. Copyright remains with the author(s).

Max Planck Institute for Tax Law and Public Finance

Marstallplatz 1

D-80539 Munich

Tel: $\quad+498924246-0$

Fax: $\quad+498924246-501$

E-mail: ssrn@tax.mpg.de

http://www.tax.mpg.de 


\title{
Sequential Majoritarian Blotto Games*
}

\author{
Tilman Klumpp ${ }^{\dagger} \quad$ Kai A. Konrad ${ }^{\ddagger}$
}

May 2018

\begin{abstract}
We study Colonel Blotto games with sequential battles and a majoritarian objective. For a large class of contest success functions, the equilibrium is unique and characterized by an even split: Each battle that is reached before one of the players wins a majority of battles is allocated the same amount of resources from the player's overall budget. As a consequence, a player's chance of winning any particular battle is independent of the battlefield and of the number of victories and losses the player accumulated in prior battles. This result is in stark contrast to equilibrium behavior in sequential contests that do not involve either fixed budgets or a majoritarian objective. We also consider the equilibrium choice of an overall budget. For many contest success functions, if the sequence of battles is long enough the payoff structure in this extended games resembles an all-pay auction without noise.
\end{abstract}

Keywords: Blotto games; dynamic battles; multi-battle contest; all-pay auctions; sequential elections.

JEL codes: D72; D74.

\section{Introduction}

We study a structure that accounts for many of the generic features of repeated conflict, the sequential majoritarian Blotto contest. Two players sequentially interact in a series of battles. Victory in each battle is decided stochastically as a function of the players'

${ }^{*}$ We thank Raphael Boleslavsky and audiences at the 2017 SAET and 2017 CPEG conferences for very helpful comments. Tilman Klumpp acknowledges financial support from the Social Sciences and Humanities Research Council of Canada (grant IG-435-2015-1397). Part of this work was completed while Tilman Klumpp was visiting the Max Planck Institute for Tax Law and Public Finance, and he thanks the members of the Institute for their warm hospitality.

${ }^{\dagger}$ Department of Economics, University of Alberta. 8-14 Tory Building, Edmonton, AB, Canada T6G 2H4. E-mail: klumpp@ualberta.ca.

${ }^{\ddagger}$ Max Planck Institute for Tax Law and Public Finance, Marstallplatz 1, D-80539 Munich, Germany. E-mail: Kai.Konrad@tax.mpg.de. 
investments of resources in this battle. A majoritarian rule applies: The player who first wins a given minimum number of battles wins the contest. Furthermore, as in a Colonel Blotto game, once the contest has started, the overall amount of resources that can be used by a player is given and cannot be augmented or reduced at a later stage, and unused resources have no scrap value outside the contest.

At any given stage of this game, players decide how much of their remaining budgets to invest trying to win the current battle, and how much to save for possible later battles. The dynamics of this interaction are not obvious. Resources tend to be more valuable at later stages of the game - in the extreme, the outcome of the final battle determines who wins the game, and players may want to preserve resources for such critical contingencies. However, the conflict may end early if one contestant wins a sufficiently large number of battles, and preserving resources for later battles is wasteful if the conflict is already decided before these later battles are reached. We find that these two forces balance each other precisely: Each battle that is reached is allocated the same share of resources from a player's overall budget as is any other battle. In every subgame perfect equilibrium, players invest constant amounts of resources in all battles in the subgame perfect equilibrium (except possibly after contingencies that are not on the equilibrium path).

The players' resource budgets with which they enter the game will be exogenous in some applications. However, in other instances players may be able to build up a stock of resources prior to the Blotto contest, which they can then deplete over the course of the contest. To study such cases, we include a costly resource build-up stage in an extended version of our model. We show that, if competitors are ex ante symmetric, virtually all rents may be dissipated in equilibrium if the sequence of battles in the Blotto game is long enough. That is, the total cost each player spends to establish his initial budget equals exactly the player's expected value of participating in the contest.

There are many instances in which competition takes the form of temporally staggered battles and where the overall resources at the contestants' disposition are fixed (more precisely: even though overall resources may be chosen by the players before the contest, they cannot be adjusted once the first battle begins).

- In military confrontations - especially rapid ones that have the features of a blitzkrieg - commanders may not have the opportunity to replenish their troops and equipment after each battle. In these cases, war leaders must allocate fixed military resources to a sequence of interactions that take place over a short period of time.

- Examples of sequential majoritarian Blotto games abound in sports. They include tennis or volleyball matches, match play golf tournaments, or best-of-five/seven playoff series in the North American professional baseball, basketball, and ice hockey leagues. A tennis player, for instance, may build up his physical strength in a long 
training period before competition. However, when standing on the court in a given match, his energy reserves will be given. He needs to allocate these reserves wisely during the match, but how much energy remains unused after the match is over is not particularly relevant.

- An important institutional example that has received considerable interest is the presidential primary elections in the United States. Presidential primaries consist of a well-structured series of elections in which candidates compete for delegates at their party's nominating convention. As we discuss below, there are several reasons to believe that the overall amount resources that primary candidates can mobilize are limited. Thus, primary candidates face a trade-off between investing campaign resources in an early battles and reserving capaign resources for a later battle.

- Organizations often allocate resources in a lumpy way to their members, who must decide on the day-to-day spending of these resources. For example, business firms might endow their marketing departments with annual budgets to run a series of marketing campaigns, or they may provide research budgets to their R\&D departments which these must allocate to a series of $R \& D$ battles with rival firms.

In all of these applications, competition is temporally staggered and budget constrained. Our results imply that the bare bones of this competition structure, coupled with a majoritarian objective, drives an even allocation of resources across battles.

This finding contrasts sharply with results that apply to sequential majoritarian contests in which the constestants' budgets can be adjusted prior to each battle (Klumpp and Polborn 2006; Konrad and Kovenock 2009; Gelder 2014). In such games, players invest more resources into early battles ("front-loading") and, generally, escalate their resource expenditures towards symmetric and more decisive states. Furthermore, winning early battles endogeneously increases the probability of winning later battles ("momentum"). What our findings suggest, then, is that front-loading, escalation, and momentum should be less pronounced if tight limits are placed on single players' overall budgets.

In military campaigns, for example, resource limits are likely to be tighter if the overall campaign is of shorter duration. Konrad (2017) discusses such dynamic conflicts, contrasting Alexander the Great's military campaign in Asia, which lasted over than ten years, and Napoleon's campaign against Russia, which lasted less than six months. In sports contests, the tradeoff between effort expended in consecutive battles should be more pronounced if the intervals for physical recovery between battles are relatively short. ${ }^{1}$ In

\footnotetext{
${ }^{1}$ For example, a tennis player's effort budget is likely fixed during a match, as the time to recover between sets is very short. On the other hand, there is typically sufficient time to "recharge" prior to the next game of a same tournament. Empirical studies of sports contests have produced mixed evidence on whether strategic momentum effects exist, or whether players front-load their efforts, in such competitions (Ferrall and Smith 1999; Malueg and Yates 2010). Our results suggest that one potential factor that affects both is the extent to which athletic resources budgets are fixed during the competition.
} 
political campaigns, a fixed budget may apply for several reasons. First, jurisdictions that provide public funding often do so in form of a fixed, lump-sum payment. ${ }^{2}$ Moreover, funds that are not spent during an election campaign may have few alternative uses. ${ }^{3}$ Finally, a candidate's resources are also fixed if competition takes the form of pledges of political favors to voters, as the aggregate of such political favors may be exogenously given. ${ }^{4}$

Static contests with fixed budgets are well studied, with the first formal analysis of the Colonel Blotto game dating back to Borel (1921). A number of important contributions in the area have been made in recent years (Kvasov 2007; Roberson 2006; Roberson and Kvasov 2012). Kovenock and Roberson (2010) review this literature and provide a systematic classification of Blotto games along several dimensions, including the contest success function that describes how the players' efforts in a given battlefield determine the outcome of the respective battle, and the players' objective functions that aggregates the outcomes of individual battles into final payoffs. Dynamic contests with fixed budgets are less well understood, but some results are available for specific cases. ${ }^{5}$ Our framework is very general in comparison to this earlier work: Players meet sequentially in a best-of- $N$ contest for arbitrary odd $N$. Players are subject to an aggregate resource constraint, but might choose their budget prior to the first battle. While the outcomes of individual battles are noisy, no specific parametric contest success function (e.g., the Tullock function) is assumed - we impose only mild conditions on the relationship between resource investments and the outcomes of individual battles.

We proceed as follows. In Section 2 we develop our sequential majoritarian Blotto framework and state our main equilibrium chracterization result, which is proven in Section 3. Section 4 examines the comparative statics of the outcome of the Blotto game,

\footnotetext{
${ }^{2}$ Candidates who accept public financing in American elections are barred from raising private funds, and are thus limited to the amount received from the state. By law, the state cannot adjust this payment in response to other candidates' spending decisions (see Klumpp et al. 2015), resulting in a fixed budget for candidates that accept public funding.

${ }^{3}$ In the U.S., unused campaign funds must be either returned to donors or saved for future election runs by the same candidate for the same office. They cannot be used by candidates for personal consumption, to support a campaign for a different office, or be forwarded to another candidate.

${ }^{4}$ Taylor (2010) argues that candidates may promise federal procurement dollars in order to secure political support in individual states. Since the total amount of such "pork" that can be pledged during a campaign is likely finite, it is best described by a fixed overall budget.

${ }^{5}$ Deck and Sheremeta (2012) consider a sequence of all-pay auctions with fixed total resources and an asymmetric objective: One player wins the game if he wins a single auction, while the opponent must win all auctions. Rinott et al. (2012) develop a model in which two teams must allocate fixed resources to their members (gladiators) who face each other in pairwise Tullock contests. The winning gladiator of the first battle faces off against the next member of the opposing team, and so on, until one team has lost all gladiators. Sela and Erez (2013) examine a Blotto model in which players maximize the number of battle victories, rather than a majoritarian objective, and each battle is a symmetric Tullock contest. Similar to the equilibrium in flexible budget models, players invest more in early battles than in later ones. Konrad (2017) studies resource carryovers between sequential battles, using a symmetric Tullock contest success function and a maximum of three battles.
} 
and its asymptotic properties, as the number of battlefields changes. Section 5 uses these results to examine an enhanced game in which each player's budget is endogenous. Section 6 concludes.

\section{Sequential Majoritarian Blotto Games}

\section{$2.1 \quad$ The contest}

We consider a Blotto tournament with players $A$ and $B$ and an odd number $N$ of battlefields, fought in sequence. Battle $N$ is fought first, battle $N-1$ is fought second, and so on, with the final battle being battle 1 . The player who first wins $n=(N+1) / 2$ battles wins the tournament. At this point, the game ends and no further battles are fought.

Players are endowed with initial resources $\bar{a}$ and $\bar{b}$ which they can invest in the battles to influence their chance of success in each battle. A player cannot spend more than his initial resources in total, and in each battle he cannot use more than the difference between his initial resources and the resources already spent. Each player's objective is to allocate his resources to battles in a consecutive way that maximizes his chance of winning a majority of battles. Players observe the outcome of each battle and the opponent's remaining resources before making simultaneous investment decisions into the next battle. (Note that observing the opponent's remaining resources before each battle is equivalent to observing the opponent's investment made into the previous battle.) Unused resources at the end of the game have no value.

The outcome of each battle is governed by a contest success function (CSF)

$$
p:[0, \infty)^{2} \rightarrow[0,1]
$$

where $p(x, y)$ is the probability that $A$ wins the battle if $A$ spends resources $x$, and $B$ spends resources $y$, on the given battle. The probability that $B$ wins the battle is $1-p(x, y)$. We maintain the following assumptions on the contest success function. $p$ is continuous everywhere except possibly at $(0,0)$. $p$ is twice differentiable on $\mathbb{R}^{2} \backslash(0,0)$ with

$p_{x} \geq 0, p_{x x} \leq 0, p_{y} \leq 0, p_{y y} \geq 0$, and these inequalities are strict at all $(x, y) \gg(0,0)$. We do not require $p$ to be symmetric, i.e., we do not assume that $1-p(x, y)=p(y, x)$.

In addition to the above assumptions, we impose the following condition:

$$
-\frac{p_{x x}(x, y)}{p_{x}(x, y)}>\frac{p_{x}(x, y)}{1-p(x, y)} \quad \text { and }-\frac{p_{y y}(x, y)}{p_{y}(x, y)}>-\frac{p_{y}(x, y)}{p(x, y)} \quad \forall(x, y) \gg(0,0) \text {. }
$$

Condition (1) requires the probability of success to be sufficiently concave in a player's own effort. The left-hand side of each inequality in (1) is a curvature measure akin to the Arrow-Pratt measure of risk aversion. The right-hand side is a hazard rate. 
Loosely speaking, it represents the chance that a marginal unit of effort results in victory conditional on not having won the battle with the effort already invested.

Many commonly used CSFs satisfy our assumptions, including all functions of the form

$$
p(x, y)=\frac{f(x)}{f(x)+g(y)},
$$

where $f, g \geq 0$ are twice differentiable, strictly increasing, and weakly concave functions. In particular, the popular Tullock (1980) lottery function

$$
p^{\text {Tullock }}(x, y)=\left\{\begin{array}{cl}
x /(x+y) & \text { if } x+y>0 \\
1 / 2 & \text { if } x+y=0
\end{array}\right.
$$

fits in our model, as do most of the variants of the Tullock function that have been explored in the literature.

\subsection{Strategies and solution concept}

In principle, a strategy for a player prescribes, for every battle and every history of spending decisions and outcomes in previous battles, an investment into the current battle. We restrict our attention to Markovian strategies, which depend on the game's history only through the total number of victories that $A$ and $B$ have accumulated and the resources the players have remaining in any battle. ${ }^{6}$

To formalize such strategies, we define a state of the tournament to be a pair $(i, j)$ such that $i, j \geq 0$ and $1 \leq i+j \leq 2 n$. This indicates that $A$ needs to win $i$ battles to win the tournament, and $B$ needs to win $j$ battles to win the tournament. If player $A$ wins a battle, $i$ is reduced by one, and if $B$ wins a battle, $j$ is reduced by one. The initial state is $(n, n)$. States $(i, j)$ with $i=0$ or $j=0$ are terminal states. At these states, one player has won the tournament and no further decisions are made.

States with $i, j \geq 1$ are non-terminal states, at which players must decide how much to invest in the current battle. An investment function for player $A$ at non-terminal state $(i, j)$ is a function

$$
\alpha_{i, j}:[0, \bar{a}] \times[0, \bar{b}] \rightarrow[0, \bar{a}] \text { s.t. } \alpha_{i, j}(a, b) \leq a
$$

This means that $\alpha_{i, j}(a, b)$ is the investment $A$ makes into the battle at state $(i, j)$ if $A$ 's remaining resources are $a$ and $B$ 's remaining resources are $b$. An investment function for

\footnotetext{
${ }^{6}$ These are the relevant information sets, as the game is of complete information and players' maximize the probability of winning a majority of battles given fixed resources. Payoffs do not directly depend on which specific battles a player wins, or on how the player's resources were allocated to specific battles. At the expense of additional notation, one can extend our analysis to non-Markovian strategies (which condition on full histories) and obtain the same results.
} 
player $B$ at $(i, j)$ is similarly defined as

$$
\beta_{i, j}:[0, \bar{a}] \times[0, \bar{b}] \rightarrow[0, \bar{b}] \text { s.t. } \beta_{i, j}(a, b) \leq b .
$$

A (pure) continuation strategy at state $(i, j)$ is then a collection of investment functions at $(i, j)$ and every possible non-terminal state that can be reached from state $(i, j)$ :

$$
\begin{aligned}
\sigma_{i, j}^{A} & =\left\{\alpha_{i^{\prime}, j^{\prime}}(\cdot):(1,1) \leq\left(i^{\prime}, j^{\prime}\right) \leq(i, j)\right\}, \\
\sigma_{i, j}^{B} & =\left\{\beta_{i^{\prime}, j^{\prime}}(\cdot):(1,1) \leq\left(i^{\prime}, j^{\prime}\right) \leq(i, j)\right\} .
\end{aligned}
$$

A (pure) strategy is a continuation strategy at the initial state $(n, n)$, that is, a collection of investment functions for every state of the game. ${ }^{7}$

Each relevant subgame of the tournament originates at an information set $(i, j ; a, b)$, consisting of a state $(i, j)$ and a pair of remaining budgets $(a, b)$. Given $(i, j ; a, b)$ and pair of continuation strategies $\left(\sigma_{i, j}^{A}, \sigma_{i, j}^{B}\right)$, one can compute the probability with which each player wins the tournament, starting at $(i, j ; a, b)$. Continuation strategy $\sigma_{i, j}^{A}$ is a best response to $\sigma_{i, j}^{B}$ at state $(i, j)$ if, for all $a \in[0, \bar{a}]$ and $b \in[0, \bar{b}]$, player $A$ 's probability of winning in subgame $(i, j ; a, b)$ is maximized if he uses strategy $\sigma_{i, j}^{A}$, conditional on $B$ using $\sigma_{i, j}^{B}$. Player $B$ 's best responses are defined similarly.

A pair $\left(\sigma_{i, j}^{A}, \sigma_{i, j}^{B}\right)$ of mutual best responses is a (pure strategy) continuation Nash equilibrium at $(i, j)$. A (pure strategy) subgame perfect equilibrium is a profile of strategies $\left(\sigma^{A}, \sigma^{B}\right)$ such, for each non-terminal state $(i, j)$ the associated profile of continuation strategies $\left(\sigma_{i, j}^{A}, \sigma_{i, j}^{B}\right)$ is a continuation Nash equilibrium at $(i, j)$.

\subsection{Equilibrium characterization}

We now present our main equilibrium characterization result. The following definition will be central:

Definition 1. For player $A$, continuation strategy $\sigma_{i, j}^{A}$ at state $(i, j)$ is an even-split continuation strategy if

$$
b>0 \Rightarrow \alpha_{i^{\prime}, j^{\prime}}(a, b)=\frac{a}{i^{\prime}+j^{\prime}-1} \forall\left(i^{\prime}, j^{\prime}\right) \text { s.t. }(1,1) \leq\left(i^{\prime}, j^{\prime}\right) \leq(i, j) .
$$

For $B$, the definition is analogous. An even-split strategy is an even-split continuation strategy at the initial state $(n, n)$.

\footnotetext{
${ }^{7} \mathrm{As}$ is common in extensive form games, to facilitate backward induction a player's strategy prescribes actions even for information sets that are not reached under this strategy. For example, if a player exhausts his entire budget in one battle he cannot invest a positive amount in the next. A strategy must still specify how much the player would invest in that battle for any amount of remaining resources.
} 
A player who uses an even-split strategy allocates his budget evenly and unconditionally across battles, as long as his opponent has positive resources remaining. For example, at the first battle $A$ invests $\bar{a} / N$ and saves the remainder $\bar{a}-\bar{a} / N$ for the next round. At the second battle he spends $(\bar{a}-\bar{a} / N) /(N-1)=\bar{a} / N$ and saves the remainder $\bar{a}-2 \bar{a} / N$ for the third round, where he invests $(\bar{a}-2 \bar{a} / N) /(N-2)=\bar{a} / N$, and so on. The player does not make this investment dependent on how many battles each player has already won, or on the amount the opponent spends in each battle.

If both players use even-split strategies, player $A$ wins each individual battle with probability $p(\bar{a} / N, \bar{b} / N)$, and $B$ wins each individual battle with probability $1-p(\bar{a} / N, \bar{b} / N)$. Our main result is that this is the unique outcome of the sequential majoritarian Blotto game.

Proposition 1. A pure strategy subgame perfect equilibrium exists in which both players use even-split strategies. Furthermore, in every subgame perfect equilibrium, both players use only even-split strategies.

To understand the second part of the result, note that an even-split strategy, as defined above, does not restrict a player's spending pattern once an opponent has run out of resources. This event will not occur in any equilibrium of our model; however, if (out of equilibrium) a subgame were reached in which one player has zero resources remaining, the other player could have multiple best responses. For example, consider the Tullock function in (3), and imagine that player $B$ has zero resources remaining at some state $(i, j)$, while $A$ has a positive resources remaining. $A$ can win the tournament with probability one by allocating a positive amount of resources to any $i$ of the remaining $i+j-1$ battles. In particular, $A$ does not have to allocate his resources evenly across all remaining battles (although this would also guarantee victory). Thus, for some CSFs the game can have multiple equilibria. However, in all of these equilibria, observed spending along the equilibrium path is the same - namely, players divide their resources evenly across battles.

\subsection{Discussion}

Proposition 1 implies that equilibrium behavior in sequential majoritarian Blotto games is markedly different from that in sequential majoritarian non-Blotto contests (Klumpp and Polborn 2006; Konrad and Kovenock 2009), or that in sequential non-majoritarian Blotto contests (Sela and Erez 2013). When the players reach state $(i, j)$ they plan for the longest possible path to final victory or defeat, which consists of $i+j-1$ battles, and allocate equal shares of their remaining budgets to each of these battles. Thus, in equilibrium, player $A$ invests $\bar{a} / N$ in every battle and $B$ invests $\bar{b} / N$ in every battle, independent of which battle it is. Players will not revise their plans in response to winning or losing any particular battle, which means that winning or losing a battle 
does not create momentum. While the player who loses the first battle will now win the tournament with a smaller probability, he wins the next battle with exactly the same probability as before. Moreover, no disproportionate share of resources is concentrated on early battles, nor does effort escalate as battles become more decisive.

These equilibrium dynamics of the sequential majoritarian Blotto game are surprising, because each player faces two conflicting incentives when deciding on how to allocates his resources dynamically across battles. For concreteness, consider the very first battle (state $(n, n))$ and the very last battle possible (state $(1,1)$ ). Whoever wins the last battle wins the tournament, while winning the first battle is only a small step toward ultimate victory. In other words, even if a player loses the first battle, he can make up for this by winning a later round; no such second chance exists in the last battle. This suggests that players may want to keep a larger share of their resources as a "strategic reserve" to invest in these increasingly decisive battles. However, there is a countervailing effect: Whereas the first battle takes place with certainty, later battles are reached only with some probability - and with a long sequence of battles, it is actually rather unlikely that state $(1,1)$ is reached in the course of the tournament. Since keeping strategic reserves

for battles that may never take place is costly, players may also prefer to not "waste" resources, and instead invest a larger share of their budgets early.

Thus, both players must balance the increased decisiveness of late battles against the decreased likelihood of reaching these battles. What Proposition 1 means, then, is that these two forces cancel each other exactly in equilibrium. Note that this is true for all contest success functions that satisfy our assumptions, and is not a knife-edge result driven by any particular functional form or parameterization of $p$. Instead, it is the specific combination of fixed resource budgets and the majoritarian objective we consider that generates the even-split equilibrium (within the class of CSFs we consider).

\section{Proof of the Main Result}

In this Section we prove Proposition 1. We first establish existence of an even-split equilibrium, and then show that every equilibrium must be in even-split strategies.

\subsection{Existence}

For $r \in\{1, \ldots, N\}$, let the set of possible states when there are exactly $r$ battles remaining be denoted by

$$
T(r)=\{(i, j) \geq(1,1): i+j-1=r\} .
$$

The proof is by induction on the number of battles remaining. Take $2 \leq r \leq N$. Suppose that at all $(i, j) \in T(r-1)$, it is a continuation Nash equilibrium for both players to use even-split continuation strategies. We will show that a pair of even-split strategies 
is a continuation Nash equilibrium at all $(i, j) \in T(r)$. Because $T(1)=\{(1,1)\}$, and continuation strategies at state $(1,1)$ are even-split (there is exactly one battle remaining and it is clearly optimal to invest any remaining resources in this battle), the result follows.

Fix $2 \leq r \leq N$. Suppose that at all $(i, j) \in T(r-1)$, an even-split continuation equilibrium exists and is played if $(i, j)$ is reached. Now fix a state $\left(i_{0}, j_{0}\right) \in T(r)$ for battle $r$. Suppose that $B$ plays an even-split continuation strategy also at $\left(i_{0}, j_{0}\right)$. (The argument is the same when the roles of the players reversed, and omitted.) Let $\left(a_{0}, b_{0}\right) \gg(0,0)$ denote the players' remaining budgets at $\left(i_{0}, j_{0}\right)$. Since $B$ plays an even-split continuation strategy, $B$ spends $\gamma=b_{0} / r$ in all battles $r, r-1, r-2, \ldots$, regardless of the states of these battles and regardless of $A$ 's spending in these battles. ${ }^{8}$ Since $\gamma>0, A$ 's probability of winning the tournament is continuous in the resources that $A$ allocates to each battle, and since the set of all such allocations is compact, $A$ will have a best response at $\left(i_{0}, j_{0}\right)$. Furthermore, if $A$ allocates $x_{0} \in\left[0, a_{0}\right]$ to battle $r$ at state $\left(i_{0}, j_{0}\right)$, he will have resources $a_{0}-x_{0}$ remaining when he reaches battle $r-1$. By the induction hypothesis, $A$ will then allocate $\left(a_{0}-x_{0}\right) /(r-1)$ to battles $r-1, r-2, \ldots$. We will show that $x_{0}=a_{0} / r$ is $A$ 's optimal investment at state $\left(i_{0}, j_{0}\right)$.

Our approach is the following: If $x_{0} \neq a_{0} / r$, player $A$ invests different amounts in battles $r$ and $r-1$. We will show that $A$ can then improve his chance of winning by equalizing spending in battles $r$ and $r-1$, while still allocating $\left(a_{0}-x_{0}\right) /(r-1)$ to battles $r-2, r-3, \ldots$. Since this reallocation is possible whenever $x_{0} \neq a_{0} / r$, we conclude that $A$ 's best response at $\left(i_{0}, j_{0}\right)$ is to use the even-split continuation strategy. ${ }^{9}$ To establish the profitability of this deviation, we proceed in three steps. In Step 1, we establish that the reallocation strictly increases $A$ 's chance of winning both battle $r$ and $r-1$, and strictly decreases $A$ 's chance of losing both battles. In Step 2, we show that this must increase $A$ 's overall chance of winning the tournament at state $\left(i_{0}, j_{0}\right)$, provided that $A$ did not spend his entire budget $a_{0}$ at state $\left(i_{0}, j_{0}\right)$; that is, we assume that $x_{0}<a_{0}$. In Step 3, finally, we show that $x_{0}=a_{0}$ cannot be a best response to $B$ 's even-split strategy.

Throughout, we assume that $i_{0}>1$ and $j_{0}>1$. The cases where $i_{0}=1$ or $j_{0}=1$ are similar and in the Appendix.

\footnotetext{
${ }^{8}$ Note that battles $r-1, r-2, \ldots$ may not take place. In general, when we say "spend in battle X" we mean "plan to spend in battle X, if battle X takes place."

${ }^{9}$ Note that, following the reallocation, $A$ no longer plays an even-split continuation strategy when he reaches battle $r-1$. (He now spends a different amount in battle $r-1$ and $r-2$.) This is not a contradiction to our induction hypothesis, as we do not claim that the new continuation strategy is optimal. The resource shift only establishes that the previous strategy (which was to spend one amount in battle $r$ and a different amount in battles $r-1, r-2, \ldots$ ) was not optimal.
} 
Step 1. Consider the two consecutive battles $r$ and $r-1$. Define

$$
\bar{x} \equiv \frac{x_{0}+\left(a_{0}-x_{0}\right) /(r-1)}{2}
$$

to be the the average amount spent by $A$ in battles $r$ and $r-1$, and set $h \equiv\left|\bar{x}-x_{0}\right|$. Note that $0 \leq h \leq \bar{x}$, and $h=0$ iff $\bar{x}=x_{0}$, or equivalently, $x_{0}=a_{0} / r=\left(a_{0}-x_{0}\right) /(r-1)$.

The probability that $A$ wins both battles is

$$
P(h)=p(\bar{x}+h, \gamma) p(\bar{x}-h, \gamma)
$$

$P$ is continuous and differentiable at all $h \in[0, \bar{x}]$, with

$$
P^{\prime}(h)=p_{x}(\bar{x}+h, \gamma) p(\bar{x}-h, \gamma)-p(\bar{x}+h, \gamma) p_{x}(\bar{x}-h, \gamma)
$$

This term is negative if and only if

$$
\frac{p_{x}(\bar{x}+h, \gamma)}{p(\bar{x}+h, \gamma)}<\frac{p_{x}(\bar{x}-h, \gamma)}{p(\bar{x}-h, \gamma)}
$$

Because $p_{x}>0$ and $p_{x x}<0$ on $\mathbb{R}_{++}^{2}$, inequality (4) holds and $P^{\prime}(h)<0$ for all $h \in[0, \bar{x}]$.

Similarly, the probability that $A$ loses both battles is

$$
Q(h)=[1-p(\bar{x}+h, \gamma)][1-p(\bar{x}-h, \gamma)] .
$$

$Q$ is continuous and differentiable at all $h \in[0, \bar{x}]$, with

$$
Q^{\prime}(h)=-p_{x}(\bar{x}+h, \gamma)[1-p(\bar{x}-h, \gamma)]+p_{x}(\bar{x}-h, \gamma)[1-p(\bar{x}+h, \gamma)]
$$

This term is positive if and only if

$$
\frac{p_{x}(\bar{x}+h, \gamma)}{1-p(\bar{x}+h, \gamma)}<\frac{p_{x}(\bar{x}-h, \gamma)}{1-p(\bar{x}-h, \gamma)}
$$

Using (1), condition (5) can be shown to hold ${ }^{10}$ so that $Q^{\prime}(h)>0$ for all $h \in[0, \bar{x}]$.

Step 2. Next, consider what happens after battle $r$ and battle $r-1$ are over. The tournament will be in one of the following three states: If $A$ won both battles, then $\left(i_{0}-2, j_{0}\right)$; if $A$ won one battle and lost the other, then $\left(i_{0}-1, j_{0}-1\right)$; and if $A$ lost both battles, then $\left(i_{0}, j_{0}-2\right)$. Let

$$
Z=\left\{\left(i_{0}-2, j_{0}\right),\left(i_{0}-1, j_{0}-1\right),\left(i_{0}, j_{0}-2\right)\right\}
$$

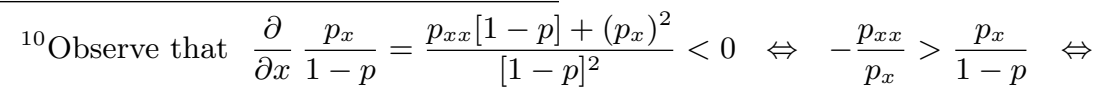


denote the set of these states.

Let $v_{i, j}$ denote the probability that $A$ wins the tournament, conditional on reaching state $(i, j) \in Z$. If $(i, j)$ is a terminal state, then $v_{i, j}=1$ if $i=0$ and $v_{i, j}=0$ if $j=0$. If $(i, j) \in Z$ is not a terminal state, then $(i, j) \in T(r-2)$, and $A$ wins the tournament if he wins $i$ of the remaining $r-2$ battles before $B$ wins $j$ battles. Since player $A$ invests $\left(a_{0}-x_{0}\right) /(r-1)$, and $B$ invests $\gamma$, into each of these remaining battles, $A$ wins each battle with probability

$$
\rho=p\left(\left(a_{0}-x_{0}\right) /(r-1), \gamma\right)
$$

Because $\gamma>0$, our assumptions in $p$ imply that $\rho<1$. Assume now that $x_{0}<a_{0}$; this implies that $\left(a_{0}-x_{0}\right) /(r-1)>0$ and hence $\rho>0$. Therefore, the probability with which $A$ wins the tournament, conditional on reaching a non-terminal state $(i, j) \in Z$, can be written as follows: ${ }^{11}$

$$
v_{i, j}=\sum_{k=0}^{j-1}\left(\begin{array}{c}
i-1+k \\
k
\end{array}\right) \rho^{i}(1-\rho)^{k} \in(0,1) .
$$

Moreover, $v_{i, j}>v_{i+1, j-1}$ for all $(i, j)$ with $(i, j) \geq(0,1){ }^{12}$

The states in the set $Z$ can hence be ordered according to $A$ 's probability of winning the tournament as follows:

$$
1 \geq v_{i_{0}-2, j_{0}}>v_{i_{0}-1, j_{0}-1}>v_{i_{0}, j_{0}-2} \geq 0
$$

Now go back to state $\left(i_{0}, j_{0}\right)$. The probability with which $A$ wins the tournament, if he spends $\bar{x}+h$ and $\bar{x}-h$ in battles $r$ and $r-1$, is

$$
z(h)=P(h) v_{i_{0}-2, j_{0}}+[1-P(h)-Q(h)] v_{i_{0}-1, j_{0}-1}+Q(h) v_{i_{0}, j_{0}-2} .
$$

Differentiating (8) with respect to $h$

$$
z^{\prime}(h)=P^{\prime}(h)\left[v_{i_{0}-2, j_{0}}-v_{i_{0}-1, j_{0}-1}\right]+Q^{\prime}(h)\left[v_{i_{0}, j_{0}-2}-v_{i_{0}-1, j_{0}-1}\right] .
$$

Because $P^{\prime}(h)<0$ and $Q^{\prime}(h)>0$ for $h \in[0, \bar{x}]$, and using $(7)$, we have $z^{\prime}(h)<0$ for all $h \in[0, \bar{x}]$. It follows that $A$ can increase his chance of winning at $\left(i_{0}, j_{0}\right)$ by reducing $h$ - that is, by reallocating some resources across the two consecutive battles $r$ and $r-1$, from the battle in which he spends more to the one in which he spends less.

\footnotetext{
${ }^{11}$ To understand (6), note that when $A$ wins the tournament, the number of remaining battles $B$ will have won is some integer $0 \leq k \leq j-1$. Each term in the sum in (6) is the probability of a sequence of $i$ wins and $k$ losses for player $A$, for given $k$.

${ }^{12}$ This is intuitive, as winning a battle should always be preferred to losing a battle. Nevertheless, a formal proof of the inequality is in the Appendix.
} 
Step 3. It remains to be shown that $x_{0}<a_{0}$. Suppose $x_{0}=a_{0}$. Then after battle $r$ is over, player $A$ spends zero on every remaining battle. In particular, he spends zero on battles $r-2, r-3, \ldots$, and wins each of these battles with probability $\rho=p(0, \gamma)<1$. If $p(0, \gamma)>0$ or if $i_{0}=2$, Step 2 can be applied without modification to show that $A$ should shift some resources from battle $r$ to battle $r-1$ to improve his overall chance of winning the tournament at state $\left(i_{0}, j_{0}\right)$. However, if $p(0, \gamma)=0$ and $i_{0}>2$, then $v_{i, j}=0$ $\forall(i, j) \in Z$ and hence $z(h)=z^{\prime}(h)=0 \forall h$. That is, player $A$ wins the tournament with probability zero, and shifting resources from battle $r$ to $r-1$ does not improve this probability. However, consider an alternative reallocation, by which player $A$ spends any positive share of $a_{0}$ on every remaining battle. Our assumptions on $p$ imply that $A$ must win each remaining battle with a strictly positive probability, resulting in a strictly positive probability that $A$ wins the tournament. It follows that $x_{0}=a_{0}$ is not a best response to $B$ 's even-split strategy at $\left(i_{0}, j_{0}\right)$.

\subsection{Uniqueness}

While multiple subgame perfect equilibria may exist, all subgame perfect equilibria are in even-split strategies. To establish this result, we make use of the fact that Blotto games are constant-sum games and, therefore, have the following property: ${ }^{13}$

Lemma 2. Consider a two-player extensive form game with mixed strategy sets $S$ and $T$. Suppose player $A$ 's expected payoff in strategy profile $(s, t) \in S \times T$ is $\pi(s, t)$ and player $B$ 's expected payoff is $c-\pi(s, t)$, for some $c$. If the strategy profiles $\left(s_{1}, t_{1}\right)$ and $\left(s_{2}, t_{2}\right)$ are Nash equilibria of the game, so are $\left(s_{1}, t_{2}\right)$ and $\left(s_{2}, t_{1}\right)$.

Since subgame perfect equilibria are strategy profiles that induce Nash equilibria in every subgame of an extensive form game, Lemma 2 immediately extends to subgame perfect equilibria.

Let $\left(\sigma_{\text {even }}^{A}, \sigma_{\text {even }}^{B}\right)$ be a pair of even-split subgame perfect equilibrium strategies of the sequential majoritarian Blotto game (which exists, as shown above). Suppose that $\left(\sigma^{A}, \sigma^{B}\right)$ is another pure strategy subgame perfect equilibrium. By Lemma 2, the profile $\left(\sigma^{A}, \sigma_{\text {even }}^{B}\right)$ is then also a subgame perfect equilibrium. Recall that in Step 2 in Section 3.1 we showed that $z^{\prime}(h)$ is strictly negative. This implies that, if player $B$ plays an even-split strategy, then every subgame-perfect best reply by player $A$ is an even-split strategy. ${ }^{14}$ Hence, $\sigma^{A}$ must be an even-split strategy, and by reversing the roles of the players one can similarly show that $\sigma^{B}$ must be an even-split strategy.

\footnotetext{
${ }^{13}$ Lemma 2 is a well-known property of constant-sum games. However, to our knowledge all published proofs establish the property for finite constant-sum games only. For completeness, a proof of the result for games with arbitray strategy spaces (along the same lines as the standard proof) is in the Appendix.

${ }^{14} \mathrm{~A}$ subgame-perfect best reply is a strategy that is a best reply at all subgames.
} 
Next, suppose there exists a subgame perfect equilibrium in mixed strategies, i.e., at least one player randomizes over at least two pure strategies. Suppose this is player $A$, and call his mixed strategy $\xi^{A}$. By Lemma 2 , the profile $\left(\xi^{A}, \sigma_{\text {even }}^{B}\right)$ is also a subgame perfect equilibrium, which implies that every pure strategy in the support of $\xi^{A}$ is a

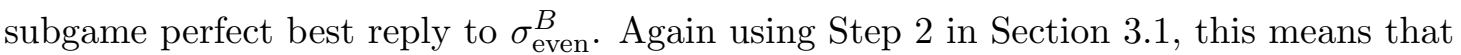
every pure strategy in the support of $\xi^{A}$ must be an even-split strategy. Therefore, any randomization in equilibrium must involve only even-split strategies.

\section{Contest Length and the Probability of Victory}

This section examines how the outcome of the sequential majoritarian Blotto game depends on the the number of battlefields.

\subsection{Individual-battle advantage}

Suppose we take a sequential $N$-battle game and increase $N$. If we do not change the initial resource budgets, the players will be forced to spread their fixed resources over an increasingly large number of battlefields. Proposition 1 establishes that players react to this change by scaling back their investments proportionally in all battles. We will show that this scaling-back generally helps the player who is more likely to win an individual battle of the contest. We call this player the "advantaged player." In some important cases the advantaged player is the player with the larger resources, but this does not always have to be the case.

Note that in every equilibrium of the $N$-battle Blotto game, player $A$ wins each individual battle with probability $p(\bar{a} / N, \bar{b} / N)$, and $B$ wins each battle with probability $1-p(\bar{a} / N, \bar{b} / N)$. To help motivate our notion of single-battle advantage, consider the following contest success function:

$$
p(x, y)=\left\{\begin{array}{cc}
\frac{x+c}{x+c+y+d} & \text { if } x+c+y+d>0, \\
1 / 2 & \text { if } x+c+y+d=0
\end{array} \quad(c, d \geq 0) .\right.
$$

If $c=d=0$, the function in (9) boils down to the Tullock lottery function (3). Note that the Tullock function is homogeneous of degree zero, so that each player's probability of winning a single battle depends on the players relative (instead of absolute) efforts in that battle. In this case, the length of the tournament has no effect on the distribution of outcomes in any individual battle - in equilibrium of the $N$-battle tournament, player $A$ wins each battle with probability $p(\bar{a} / N, \bar{b} / N)=p(\bar{a}, \bar{b})$. Thus, player $A$ has an advantage if $p(\bar{a}, \bar{b})>1 / 2$, and player $B$ has an advantage if $p(\bar{a}, \bar{b})<1 / 2$. The identity of the advantaged player depends on the players' resource budgets but not on the length of the 
contest. Moreover, if $p$ is symmetric (i.e., $p(x, y)=1-p(y, x)$ ), the advantaged player is the player who has the larger initial resource budget.

On the other hand, if $c>0$ or $d>0$ or both, $p(\bar{a} / N, \bar{b} / N)$ depends on $N$ and converges to $c /(c+d)$ as $N$ increases. As long as $N$ is sufficiently large, player $A$ has an advantage if $c>d$, while $B$ has an advantage if $c<d$. In this case, the identity of the advantaged player does not depend on $\bar{a}$ or $\bar{b}$. Moreover, if $p$ is symmetric (i.e., $c=d$ ), neither player has an advantage in the limit, regardless of the relative size of their initial resource budgets.

\subsection{An amplification result}

The preceding discussion makes clear that a player's advantage in an individual battle can depend on the player's relative resources, on the contest length, and on the shape of the contest success function. We now turn to the question how this per-battle advantage translates into relative success probabilities in the overall tournament. Recall that player $A$ wins the $N$-battle game if and only if $A$ wins $n=(N+1) / 2$ battles before player $B$ wins $n$ battles. Thus, player $A$ 's win probability can be expressed in the same way as (6), replacing $i$ and $j$ with $n$ and replacing $\rho$ with $p(\bar{a} / N, \bar{b} / N)$ :

$$
\pi(\bar{a}, \bar{b}, N) \equiv \sum_{k=0}^{n-1}\left(\begin{array}{c}
n+k-1 \\
k
\end{array}\right) p(\bar{a} / N, \bar{b} / N)^{n}(1-p(\bar{a} / N, \bar{b} / N))^{k} .
$$

Player $B$ 's probability of winning is then $1-\pi^{A}(\bar{a}, \bar{b}, N)$.

The following result provides conditions under which a large number of battlefields amplifies a player's existing individual-battle advantage.

Proposition 3. Consider a sequential majoritarian Blotto game with $N$ battlefields and initial resource endowments $\bar{a}>0$ and $\bar{b}>0$. Define $\bar{p}=\lim _{N \rightarrow \infty} p(\bar{a} / N, \bar{b} / N)$.

(a) If $\bar{p}>1 / 2(\bar{p}<1 / 2)$, player $A(B)$ has a per-battle advantage for sufficiently large $N$. As $N \rightarrow \infty$, the probability that player $A(B)$ wins the tournament converges to 1 .

If $p$ is homogeneous of degree zero and symmetric, statement (a) can be strengthened to the following:

(b) If $\bar{a}>\bar{b}(\bar{a}<\bar{b})$, player $A$ (B) has a per-battle advantage for all $N$. The probability that player $A(B)$ wins the tournament is strictly increasing in $N$, and converges to 1 as $N \rightarrow \infty$.

(c) If $\bar{a}=\bar{b}$, then neither player has an advantage, and both players win the tournament with probability $1 / 2$, for all $N$. 
Proposition 3 implies that splitting a shorter tournament into a larger number of battles tends to increases the win probability of the advantaged player and tends to decrease the win probability of the disadvantaged player. This, in turn, implies that the advantaged player prefers to lengthen the contest, and the disadvantaged player prefers to shorten it.

To gain an intuition for this result, it will be helpful to transform a player's win probability into the following form (which is also used in the proof of Proposition 3 in the Appendix):

Lemma 4. The expression in (10) is equivalent to

$$
\pi(\bar{a}, \bar{b}, N)=\sum_{k=n}^{N}\left(\begin{array}{c}
N \\
k
\end{array}\right) p(\bar{a} / N, \bar{b} / N)^{k}(1-p(\bar{a} / N, \bar{b} / N))^{N-k} .
$$

Note that (11) is simply the probability that $A$ wins more than half out of a total $N$ independent battles, assuming that all $N$ battles are fought out (instead of ending the game once the first player has won a majority of battles). The same logic as in the Condorcet Jury Theorem now applies: If winning the contest depends only on winning a majority of battles, and the outcomes of the battles are independent, then even a slightly higher probability of victory in every individual battle compounds over the course of a long tournament. In the limit, a long enough contest selects the advantaged player with certainty.

However, Proposition 3 is more than just an application of the Condorcet Jury Theorem, as the win probabilities in the individual battles are not exogenous in our context. Instead they are chosen by the players to maximize their chances of winning the $N$-battle tournament. Thus, Proposition 3 relies on the even-split nature of the equilibrium in the $N$-battle tournament, which we established in Propositions 1.

\subsection{Examples}

We conclude the section with a several examples that illustrate further aspects of the dependence of players' win probabilities on the length of the contest. The first example highlights the amplification effect in Proposition 3.

Example 1. Consider the Tullock contest success function (3) and suppose that $\bar{a}=2 \bar{b}$. Note that $p$ is homogeneous and symmetric, and since player $A$ has the larger budget, player $A$ is the advantaged player. Proposition 3 implies that in equilibrium of our multi-battle contest $A$ 's probability of winning is increasing in the length of the contest. This is illustrated by the top left panel in Figure 1: While player $A$ 's probability of winning an individual battle is $p(\bar{a} / N, \bar{b} / N)=2 / 3$ and independent of $N$, player $A$ wins the contest with probability $\pi(\bar{a}, \bar{b}, N)$, which is increasing in $N$ and converges to 1 . The 
widening gap between the solid and dotted lines $(p(\bar{a} / N, \bar{b} / N)$ vs. $\pi(\bar{a}, \bar{b}, N))$ represents the amplification effect generated by increasing the contest length.

The next three examples apply to conditions which are not covered by Proposition 3. Example 2 shows that an increase in the length of the contest does not necessarily amplify a player's single-battle advantage; Example 3 shows that the effect of increasing $N$ does not necessarily have to be monotonic; and Example 4 shows that the advantaged player's probability of winning the tournament may converge to a value strictly between $1 / 2$ and 1.

Example 1

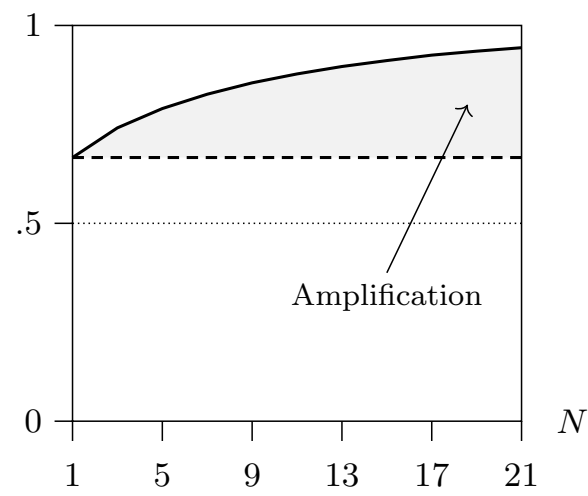

Example 3

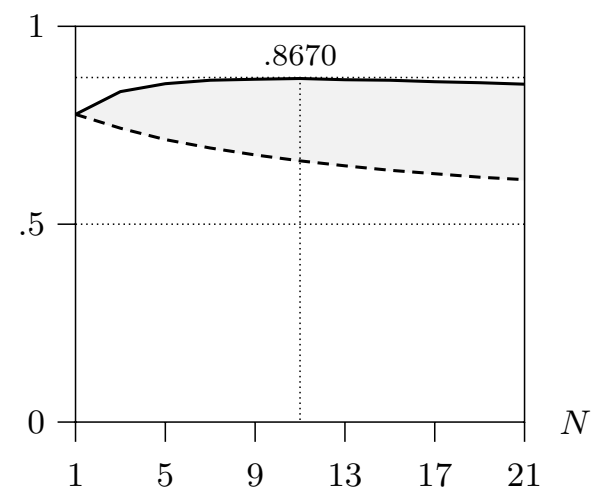

Example 2

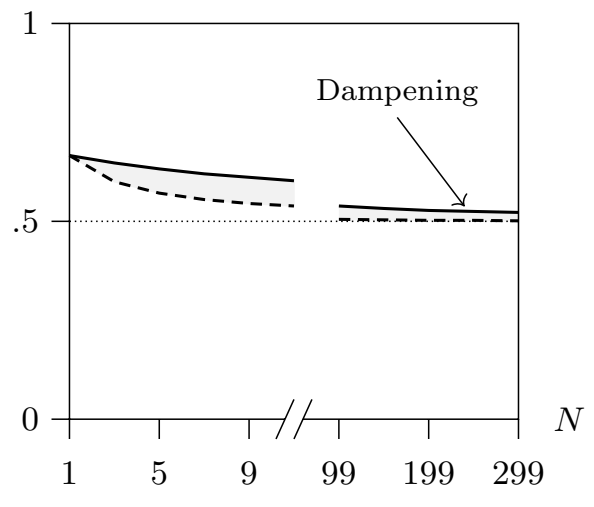

Example 4

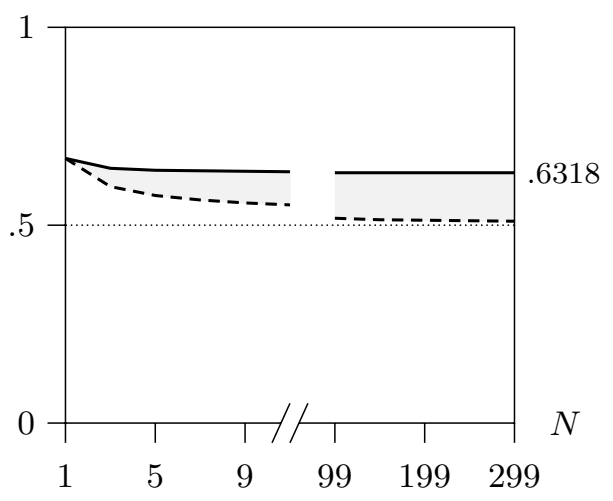

- $\pi(\bar{a}, \bar{b}, N) \quad$ (= probability that $A$ wins the $N$-battle contest)

--- $p(\bar{a} / N, \bar{b} / N) \quad$ (= probability that $A$ wins an individual battle of the $N$-battle contest)

Figure 1: Win probability as a function of contest length. 
Example 2. Consider the contest success function in (9), with $c=d=1$ :

$$
p(x, y)=\frac{x+1}{x+y+2}
$$

and suppose $\bar{a}=3$ and $\bar{b}=1 .{ }^{15}$ In the even-split equilibrium of the $N$-battle tournament, player $A$ wins each battle with probability $p(\bar{a} / N, \bar{b} / N)=(3 / N+1) /[4 / N+2]>1 / 2$, which means player $A$ has an individual-battle advantage for all $N$. Note, however, that $p(\bar{a} / N, \bar{b} / N)$ is decreasing in $N$ and converges to $1 / 2$. In contrast to Example 1 , an increase in the number of battles is now accompanied by a decrease in the advantaged player's probability of winning each battle. The top right panel in Figure 1 shows that the second effect dominates the first: As $N \rightarrow \infty$, player $A$ 's probability of winning the entire tournament decreases and converges to $1 / 2$. Thus, an increase in the number of battlefields has the effect of dampening $A$ 's advantage.

Example 3. Consider the same contest success function as in Example 2, but suppose that $\bar{a}=20$ and $\bar{b}=5$. In contrast to the previous example, an increase in the number of battles first amplifies $A$ 's per-battle advantage. The bottom left panel in Figure 1 shows that $\pi(\bar{a} / N, \bar{b} / N)$ reaches a maximum of 0.8670 at $N=11$ and begins to decrease thereafter.

Example 4. Consider the contest success function

$$
p(x, y)=\frac{1+(x-y)\left(x^{2}+y^{2}\right)^{1 / 4}}{2(x+y)}
$$

and suppose that $\bar{a}=0.25$ and $\bar{b}=0.05$. One can verify that this CSF satisfies our assumptions on $p$, including the concavity condition (1), in the relevant ranges $x \in[0,0.25]$ and $y \in[0,0.05]$. Thus, an even-split equilibrium exists. Since $p$ is symmetric but $\bar{a}>\bar{b}$, player $A$ wins each battle with probability $p(\bar{a} / N, \bar{b} / N)>1 / 2$, so player $A$ has an individual-battle advantage for all $N$. As was the case in Example 2, $p(\bar{a} / N, \bar{b} / N)$ is decreasing in $N$ and converges to $1 / 2$ as $N \rightarrow \infty$. The right panel in Figure 1 shows that, unlike in the previous example, player $A$ 's probability of winning the tournament converges to 0.6318 .

\section{Endogenous Budget Choice}

We so far considered Blotto games in which the initial budgets $(\bar{a}, \bar{b})$ were exogenously given. In many applications of our model this will not be the case, however. While a politician's financial resources may be relatively fixed during a formal election campaign,

\footnotetext{
${ }^{15}$ The contest success function is not covered by Proposition 3 (a) because $\bar{p}=1 / 2$, and it is not covered by Proposition 3 (b)-(c) because $p$ is not h.o.d. 0 .
} 
prior to the campaign candidates can raise funds to build up their "campaign war chests." While an athlete's total endurance is largely fixed during an actual competition, and must be allocated carefully across its various stages (e.g., the sets of a tennis match), between competitions athletes usually train to be in optimal shape when they compete. And while the strength of an army cannot quickly be adjusted during an armed conflict, countries can obviously adjust the size and capabilities of their militaries in the long run.

We now study these situations in an extended model where players simultaneously choose the size of their budgets at an initial fundraising stage, observe these choices, and then play the $N$-battle Blotto game. We call such games Blotto games with endogenous budgets. The fundraising stage adds an additional layer of competition to our model, and while the second stage - the sequential Blotto tournament - is still a constant-sum game, the overall model is now variable-sum.

We assume that both players attach value $V=1$ to a tournament win (compared to zero in case of losing the tournament), and that the cost of choosing budgets $\bar{a}$ and $\bar{b}$ are linear, with $C(\bar{a})=\bar{a}$ and $C(\bar{b})=\bar{b}$, respectively. For simplicity, we focus on the case where $p$ is symmetric and homogeneous of degree zero.

In the extended game player $A$ maximizes, with respect to $\bar{a} \geq 0$, the payoff function

$$
u^{A}(\bar{a}, \bar{b})=\pi(\bar{a}, \bar{b}, N) V-C(\bar{a})=\sum_{k=n}^{N}\left(\begin{array}{c}
N \\
k
\end{array}\right) p(\bar{a}, \bar{b})^{k}(1-p(\bar{a}, \bar{b}))^{N-k}-\bar{a} .
$$

Player $B$ 's payoff $u^{B}$ is defined similarly:

$$
u^{B}(\bar{a}, \bar{b})=(1-\pi(\bar{a}, \bar{b} ; N)) V-C(\bar{b})=\left[1-\sum_{k=n}^{N}\left(\begin{array}{c}
N \\
k
\end{array}\right) p(\bar{a}, \bar{b})^{k}(1-p(\bar{a}, \bar{b}))^{N-k}\right]-\bar{b} .
$$

Therefore, the extended game can be interpreted as a one-shot, variable-sum contest with linear costs and contest success function $\pi(\cdot, N)$.

Clearly, no player will choose a budget greater than $V$, as the value of competing in the second stage is at most $V$. Let us now assume that player $B$ chooses budget $\bar{b}>0$. Then $u^{A}$ is continuous in $\bar{a}$, and a value $\bar{a} \in[0, V]$ exists that maximizes (12). This budget must satisfy the first-order condition

$$
\begin{aligned}
\frac{\partial u^{A}}{\partial \bar{a}}=p_{x}(\bar{a}, b) \sum_{k=n}^{N}\left(\begin{array}{l}
N \\
k
\end{array}\right) & {\left[k p(\bar{a}, \bar{b})^{k-1}(1-p(\bar{a}, \bar{b}))^{N-k}\right.} \\
& \left.-(N-k)(1-p(\bar{a}, \bar{b}))^{N-k-1} p(\bar{a}, \bar{b})^{k}\right]-1=0 .
\end{aligned}
$$


At a symmetric pure-strategy subgame perfect equilibrium of the extended game, $\bar{a}=\bar{b}$ and thus $p(\bar{a}, \bar{a})=1 / 2$. Making these substitutions in (14) and simplifying yields

$$
p_{x}(\bar{a}, \bar{a}) \frac{1}{2^{N-1}} \sum_{k=n}^{N}\left(\begin{array}{l}
N \\
k
\end{array}\right)(2 k-N)=1 \Rightarrow p_{x}(\bar{a}, \bar{a})=4^{n-1} /\left[n\left(\begin{array}{l}
N \\
n
\end{array}\right)\right] .
$$

Let $\bar{a}^{*}(N)$ be the value for $\bar{a}$ that solves (15) for given $N$. Note that the right-hand side of (15) is decreasing in $N$. Since $p$ is homogeneous of degree zero, $p_{x}$ is homogeneous of degree -1 , so that $\bar{a}^{*}(N)$ must be increasing in $N$ : Players will raise more funds if they anticipate a longer contest. Since each player wins with probability $1 / 2$, regardless of the length of the contest, increasing the number of battlefields leads to more rent dissipation.

For an illustration, let us consider the Tullock lottery contest, given in (3). We have

$$
p_{x}^{\text {Tullock }}(\bar{a}, \bar{b})=\frac{\bar{b}}{(\bar{a}+\bar{b})^{2}} \Rightarrow p_{x}^{\text {Tullock }}(\bar{a}, \bar{a})=\frac{1}{4 \bar{a}} .
$$

Using (15) we obtain the following equilibrium budgets:

$$
\bar{a}^{*}(1)=\frac{1}{4}, \quad \bar{a}^{*}(3)=\frac{3}{8}, \quad \bar{a}^{*}(5)=\frac{15}{32} .
$$

Since players' total rent is fixed at $V=1$, the rent dissipation rate in equilibrium is $2 \bar{a}^{*}(N)$, and equals $1 / 2,3 / 4$, and $15 / 16$, respectively, for $N=1,3,5$.

For a best-of-7 Tullock contest, the solution to (15) is $\bar{a}^{*}(7)$ is $\bar{a}^{*}(7)=35 / 64>1 / 2$, which means that each player spends more than his expected share of the prize. Because a player can always choose a zero budget and guarantee at least a zero payoff, $\bar{a}^{*}(7)$ cannot be an equilibrium budget. In general, (15) describes a pure strategy equilibrium only if $N$ is sufficiently small. For large $N$, any equilibrium must involve randomization at the fundraising stage.

We do not solve for the mixed strategy equilibrium, but establish an asymptotic result for large- $N$ case. For homogeneous and symmetric $p$, Proposition 3 (b)-(c) implies that player $A$ 's probability of winning the tournament converges to

$$
\lim _{N \rightarrow \infty} \pi(\bar{a}, \bar{b}, N)=p^{\text {all-pay }}(\bar{a}, \bar{b})=\left\{\begin{array}{ccc}
1 & \text { if } & \bar{a}>\bar{b} \\
1 / 2 & \text { if } & \bar{a}=\bar{b} \\
0 & \text { if } & \bar{a}<\bar{b}
\end{array}\right.
$$

Note that this is the contest success function of a one-shot all-pay auction without noise. In the all-pay auction, a player wins with certainty if he spends even slightly more than his opponent.

Baye et al. (1996; Theorem 1, p. 293) show that the one-shot game with success function $p^{\text {all-pay }}$ and cost functions $C(\bar{a})=\bar{a}$ and $C(\bar{b})=\bar{b}$ has a unique Nash equilibrium, 
in which both players randomize their spending uniformly on $[0,1]$. In this equilibrium, each player invests $1 / 2$ on expectation; and each player makes the larger investment (and wins) with probability $1 / 2$. Thus, each player receives an expected payoff of zero, meaning all rents are dissipated.

In our model, of course, $N$ must always be finite: It does not make sense to consider a sequence of battles that never ends, because it could never be verified that one player has won a majority of battles. The question is whether the uniform equilibrium that arises in the all-pay auction approximates the first-stage equilibrium behavior in our endogenous budget game, if $N$ is large. There are, to our knowledge, no results that imply that this is the case, and we will not pursue this issue here. ${ }^{16}$

We can, however, show that uniform randomization is an $\varepsilon$-equilibrium of large $N$-battle games, and that rents are almost dissipated in these equilibria. ${ }^{17}$

Proposition 5. Let $p$ be a symmetric and h.o.d. 0 contest success function. For every $\varepsilon>0$, there exists $N^{*}$ such that the following is true for all $N>N^{*}$ : In the $N$-battle Blotto game with endogenous budgets, an $\varepsilon$-subgame perfect equilibrium exists in which the players randomize their budgets uniformly on $[0,1]$ and then play the even-split equilibrium in the Blotto game with the realized budgets. In this $\varepsilon$-equilibrium, each player's expected payoff is at most $\varepsilon$.

Proposition 5 shows that the all-pay auction without noise can be regarded as a suitable approximation of majoritarian Blotto games with endogeneous budgets, provided the contest success function that governs each individual battle of the Blotto game is symmetric and homogeneous.

However, if $p$ does not have these properties, play in the Blotto game with endogeneous budgets can be quite different from that described in Proposition 5. To illustrate this, we return to Example 2 from the previous section:

Example 2, continued. Consider again the CSF $p(x, y)=(x+1) /(x+y+2)$. As shown in Section 4.3, the probability that player $A$ wins in equilibrium of an $N$-battle

\footnotetext{
${ }^{16}$ The question whether equilibria of discontinuous games are similar to equilibria of close-by continuous games has long been of interest to game theorists. Dasgupta and Maskin (1986, p. 38) make a statement which suggests that, for the type of discontinuity present in the all-pay auction, this is the case; however, a formal result is not proven. Börgers (1991) examines the approximation of a continuous game by a sequence of discontinuous games (whereas we have a sequence of continuous games converging to a discontinuous game). Bagh (2010) studies the approximation of a discontinuous game by a sequence of continuous games, but to verify his conditions one needs to know the equilibria of the continuous games (whereas we know the equilibrium of the discontinuous game).

${ }^{17} \varepsilon$-equilibria are strategy profiles in which no player can improve his payoff by more than $\varepsilon$ when switching to another strategy. The almost-complete dissipation result can be strengthened to show that rents are fully dissipated in all mixed strategy, subgame perfect equilibria, e.g., by using the method developed in Klumpp and Polborn (2006) for simultaneous non-Blotto games (Proposition 2, p. 1083).
} 
Blotto game with this CSF converges to

$$
\lim _{N \rightarrow \infty} \pi(\bar{a}, \bar{b}, N)=\frac{1}{2} \forall \bar{a}, \bar{b} .
$$

In a contest governed by this limit function, a player's budget has no influence on the probability of winning, which means that the players will choose zero budgets. Since $p$ is continuous in $x$ and $y, \pi(\bar{a}, \bar{b}, N)$ is continuous in $\bar{a}, \bar{b}$ for all $N$, which means that convergence of $\pi(\cdot)$ to its limit is uniform. It follows that a pair of zero budgets, and zero rent dissipation, is an approximation (in the sense of $\varepsilon$-equilibrium) of the outcome in a large- $N$ Blotto game with CSF $p$ and endogeneous budgets.

\section{Conclusion}

We examined a two-player, multi-battle competition characterized by three main features: The battles are fought sequentially; the player who first wins a majority of battles wins the contest; and both players are endowed with fixed budgets that must be allocated across the battles. We called such games sequential majoritarian Blotto games. The tradeoffs the players face when deciding how much of their resources to invest in each battle are not trivial. Yet, we showed that a very simple strategy - namely, to allocate the same proportion of one's intitial resources to every battle - uniquely describes equilibrium behavior in this game. Therefore, the probability with which a given player wins each individual battle remains constant from the beginning to the end of the game.

These dynamics contrast sharply with those arising in equilibrium of sequential majoritarian non-Blotto games. In these games, players invest more resources into early battles and battles that are more decisive, and winning early battles increases the probability of winning later battles. Therefore, our analysis identifies a key aspect for whether such front-loading, escalation, and momentum effects arise - the extent to which competitors have the opportunity to adjust their resource budgets before each new battle. In Section 1 we argued that such adjustments could be difficult or impossible for certain kinds of military confrontations, for sports competitions in which the period for physical recovery between individual battles is short, and for sequential elections in which candidates are publicly funded or compete by allocating political favors. ${ }^{18}$

\footnotetext{
${ }^{18}$ Of course, resource allocation in dynamic battles may also be influenced by a number of other, context-specific factors. Consider sequential elections, such as the U.S. presidential primaries. Victory in one election can cause an inflow of additional resources to a candidate (the so-called Mathews effect; see Aldrich 1980), and it can make resources invested in the next round more effective (for example, as a result of the publicity surrounding the first win; see Brams and Davis 1982). Finally, the information and learning aspects that can emerge from sequential voting processes (e.g., Dekel and Piccione 2000; Hummel and Knight 2015) may also influence how candidates allocate their resources across battles. To isolate the role of fixed budgets, we do not consider these factors in our model. In the absence of such aspects, our analysis suggests that budget-constrained candidates allocate their budgets evenly across all battles.
} 
Even though the equilibrium resource allocation is simple, regardless of how many component battles the game contains, the dependence of a player's overall probability of victory on the number of battles is more complex. We derived sufficient conditions under which a Condorcet Jury Theorem-type effect dominates in games with a large number of battles: The player who is more likely to win any individual battle of the contest wins the overall $N$-battle game with probability one in the limit. For homogeneous and symmetric contest success functions, this is the player with the larger initial resource budget. In an extended model in which players build up their budgets prior to the Blotto game, this amplification effect implies that all rents are dissipated if the number of battles is large. Thus, either a small or a large number of battles may be socially optimal, depending on whether effort is seen as socially wasteful or socially useful (which, of course, depends on the application one has in mind).

Our results relate to a number of insights developed in the literature on elimination tournaments, which we briefly mention here. In elimination tournaments, players compete in a series of stages (e.g., quarterfinals, semifinals, finals), with the winners of one stage advancing to compete in the next (see Rosen 1986). Harbaugh and Klumpp (2005) compare a two-stage, four-player Tullock elimination tournament with fixed resource budgets to one in which players have separate budgets in each round. They show that having a fixed budget induces weaker contestants to front-load their efforts on the first round, and stronger contestants to preserve resources for the second round. They find empirical support for this prediction, exploiting a rule change in the NCAA men's basketball tournament. Gradstein and Konrad (1999) and Fu and Lu (2012) study elimination contests in which a large set of players is partitioned into subgroups. The subgroups compete internally, and their winners become the participants in the next contest stage. This process continues until the final winner is determined the last round. In both models, an increase in the number of elimination rounds effectively removes the noise that is present in each battle, thereby raising the marginal benefit of effort. This mirrors our result that repetition of noisy interactions can "wash out" noise in repeated interaction, and thereby enhance the incentives to invest in budgets.

\section{Appendix}

\section{Remaining steps in the proof of Proposition 1}

Here we complete the proof of the existence of an even-split equilibrium in the sequential majoritarian Blotto game. For the most part, the argument was developed in Section 3.1. What remains to be done is the following: 
A. Show that $v_{i, j}>v_{i+1, j-1}$ for all $(i, j) \geq(0,1)$, where $v_{i, j}$ is defined in $(6)$.

B. Repeat the same steps as in the text for the following cases: (i) $i_{0}=1$ and $j_{0}>1$; (ii) $i_{0}>1$ and $j_{0}=1$.

Part A. Since $\rho \in(0,1),(6)$ implies that

$$
v_{i, j} \in(0,1) \quad \forall(i, j) \geq(1,1), \quad v_{0, j}=1 \quad \forall j \geq 1, \quad v_{i, 0}=0 \quad \forall i \geq 1 .
$$

The proof is by induction. Fix $d \geq 1$ and suppose that

$$
v_{i, j}>v_{i+1, j-1} \quad \forall(i, j) \geq(0,1) \text { s.t. } i+j=d .
$$

Take $(i, j) \geq(0,1)$ with $i+j=d+1$. If $i \geq 1$ and $j \geq 2$, write

$$
v_{i, j}=\rho v_{i-1, j}+(1-\rho) v_{i, j-1} \quad \text { and } \quad v_{i+1, j-1}=\rho v_{i, j-1}+(1-\rho) v_{i+1, j-2} .
$$

By (18) we have

$$
v_{i, j}-v_{i+1, j-1}=\rho\left(v_{i-1, j}-v_{i, j-1}\right)+(1-\rho)\left(v_{i, j-1}-v_{i+1, j-2}\right)>0 .
$$

If $i=0$ then $j \geq 2$ and (17) implies

$$
v_{0, j}=1, v_{1, j-1} \in(0,1) \Rightarrow v_{0, j}-v_{1, j-1}>0 .
$$

Similarly, if $j=1$ then $i \geq 1(17)$ implies

$$
v_{i, 1} \in(0,1), v_{i+1,0}=0 \Rightarrow v_{i, 1}-v_{i+1,0}>0 .
$$

Thus, for all $(i, j) \geq(0,1)$ with $i+j=d+1$, we have $v_{i, j}>v_{i+1, j-1}$. Now note that (18) is clearly true for $d=1\left(v_{0,1}=1>0=v_{1,0}\right)$, and the result follows.

Part B. First consider case (i). Since $i_{0}=1$, once player $A$ wins battle $r$ he has won the tournament. Therefore, the term $P(h)$ (i.e., the probability that $A$ wins battle $r$ and battle $r-1$ ) is not defined. The term $Q(h)$ (i.e., the probability that $A$ loses battle $r$ and battle $r-1$ ) is defined as before and strictly increasing in $h$. After battle $r$ and (if necessary) battle $r-1$ are over, the tournament will be in some state

$$
(i, j) \in Z=\left\{\left(0, j_{0}\right),\left(0, j_{0}-1\right),\left(1, j_{0}-2\right)\right\}
$$


For $\gamma>0$ and $x_{0} \in\left[0, a_{0}\right]$, the probabilities $v_{i, j}$ that player $A$ wins the tournament at $(i, j) \in Z$ can therefore be ranked as follows:

$$
1=v_{0, j_{0}}=v_{0, j_{0}-1}>v_{1, j_{0}-2} \geq 0
$$

Going back to state $\left(i_{0}, j_{0}\right)$, player $A$ wins the tournament with probability

$$
z(h)=1-Q(h)+Q(h) v_{1, j_{0}-2} .
$$

Note that $z^{\prime}(h)=Q^{\prime}(h)\left[v_{1, j_{0}-2}-1\right]<0$, and it follows that $A$ can increase his chance of winning the tournament by reducing $h$.

Next, consider case (ii). Since $j_{0}=1$, once player $A$ loses battle $r$ he has lost the tournament. Therefore, the term $Q(h)$ is not defined; the term $P(h)$ is defined as before and strictly decreasing in $h$. After battle $r$ and (if necessary) battle $r-1$ are over, the tournament will be in some state

$$
(i, j) \in Z=\left\{\left(i_{0}-2,1\right),\left(i_{0}-1,0\right),\left(i_{0}, 0\right)\right\} .
$$

For $\gamma>0$ and $x_{0} \in\left[0, a_{0}\right]$, the probabilities $v_{i, j}$ that player $A$ wins the tournament at $(i, j) \in Z$ can therefore be ranked as follows:

$$
1 \geq v_{i_{0}-2,1}>v_{i_{0}-1,0}=v_{i_{0}, 0}=0
$$

Going back to state $\left(i_{0}, j_{0}\right)$, player $A$ wins the tournament with probability

$$
z(h)=P(h) v_{i_{0}-2,1}
$$

Note that $z^{\prime}(h)=P^{\prime}(h) v_{i_{0}-2,1}<0$, and it follows that $A$ can increase his chance of winning the tournament by reducing $h$.

\section{Proof of Lemma 2}

In Nash equilibrium of a constant-sum game, player $A$ maximizes $\pi$ by choice of $s \in S$, and player $B$ minimizes $\pi$ by choice of $t \in T$. Let $\left(s_{1}, t_{1}\right)$ and $\left(s_{2}, t_{2}\right)$ be two Nash equilibria. Because $\left(s_{1}, t_{1}\right)$ is an equilibrium,

$$
\pi\left(s_{1}, t_{1}\right) \geq \pi\left(s_{2}, t_{1}\right) \quad \text { and } \quad \pi\left(s_{1}, t_{1}\right) \leq \pi\left(s_{1}, t_{2}\right)
$$

(otherwise player $A$ would deviate from $s_{1}$ to $s_{2}$, or player $B$ would deviate from $t_{1}$ to $\left.t_{2}\right)$. Similarly, because $\left(s_{2}, t_{2}\right)$ is an equilibrium,

$$
\pi\left(s_{2}, t_{2}\right) \geq \pi\left(s_{1}, t_{2}\right) \quad \text { and } \quad \pi\left(s_{2}, t_{2}\right) \leq \pi\left(s_{2}, t_{1}\right)
$$


Combining the four weak inequalities in (19)-(20), we get $\pi\left(s_{1}, t_{1}\right) \geq \pi\left(s_{2}, t_{1}\right) \geq$ $\pi\left(s_{2}, t_{2}\right) \geq \pi\left(s_{1}, t_{2}\right) \geq \pi\left(s_{1}, t_{1}\right)$, and it follows that

$$
\pi\left(s_{1}, t_{1}\right)=\pi\left(s_{2}, t_{1}\right)=\pi\left(s_{2}, t_{2}\right)=\pi\left(s_{1}, t_{2}\right)=\pi\left(s_{1}, t_{1}\right)
$$

By definition, $\pi\left(s_{1}, t_{1}\right)$ is the maximum payoff $A$ can obtain if $B$ uses strategy $t_{1}$. By the first equality in (21), if $A$ uses strategy $s_{2}$ against $t_{1}$ he obtains exactly this maximum payoff; hence $s_{2}$ is a best reply to $t_{1}$. Similarly, $\pi\left(s_{2}, t_{2}\right)$ is the minimum payoff that $A$ can obtain if he uses strategy $s_{2}$. By the second equality in (21), if $B$ uses strategy $t_{1}$ against $s_{2}$ then $A$ obtains exactly this minimum payoff; hence $t_{1}$ is a best reply to $s_{2}$. It follows that the profile $\left(s_{2}, t_{1}\right)$ must also be a Nash equilibrium. An analogous argument, using the third and fourth equality in $(21)$, establishes that $\left(s_{1}, t_{2}\right)$ is a Nash equilibrium.

\section{Proof of Lemma 4}

Consider a vector $\nu=\left(\nu_{1}, \nu_{2}, \ldots, \nu_{N}\right)$ of i.i.d. draws $\nu_{i} \in\{0,1\}$, where $\operatorname{Pr}\left[\nu_{i}=1\right]=\rho$. Given $\nu \in\{0,1\}^{N}$, define

$$
W_{i}(\nu)=\sum_{i^{\prime}=1}^{i} \nu_{i^{\prime}}, \quad m(\nu)=\left\{\begin{array}{cl}
\min \left\{i: W_{i}=n\right\} & \text { if } W_{N} \geq n \\
-1 & \text { otherwise }
\end{array}\right.
$$

Observe that $W_{N}(\nu) \geq n$ if and only if $m(\nu) \geq n$. Thus,

$$
\begin{aligned}
\operatorname{Pr}\left[W_{N}(\nu) \geq n\right] & =\sum_{W_{N}=n}^{N}\left(\begin{array}{c}
N \\
W_{N}
\end{array}\right) \rho^{W_{N}}(1-\rho)^{N-W_{N}} \\
& =\sum_{m=n}^{N}\left(\begin{array}{c}
m-1 \\
m-n
\end{array}\right) \rho^{n}(1-\rho)^{m-n}=\operatorname{Pr}[m(\nu) \geq n] .
\end{aligned}
$$

To understand the second sum in (23), take $m \geq n$. By definition of $m(\nu), \rho(\nu)=m$ if and only if $\left|\left\{i \leq m: \nu_{i}=1\right\}\right|=n,\left|\left\{i \leq m: \nu_{i}=0\right\}\right|=m-n$, and $\nu_{m}=1$. Each term in the sum is the probability that $\nu$ has these properties for given $m$, and we iterate over $m \in\{n, \ldots, N\}$. Now substitute $k$ for $W_{N}$ in the first sum in (23), substitute $k$ for $m-n$ in the second sum, and substitute $p(\bar{a} / N, \bar{b} / N)$ for $\rho$ in both sums, to get

$$
\begin{aligned}
& \sum_{k=n}^{N}\left(\begin{array}{c}
N \\
k
\end{array}\right) p(\bar{a} / N, \bar{b} / N)^{k}(1-p(\bar{a} / N, \bar{b} / N))^{N-k} \\
& \quad=\sum_{k=0}^{n-1}\left(\begin{array}{c}
n+k-1 \\
k
\end{array}\right) p(\bar{a} / N, \bar{b} / N)^{n}(1-p(\bar{a} / N, \bar{b} / N))^{k}
\end{aligned}
$$


These are the expressions in (11) and (10), respectively.

\section{Proof of Proposition 3}

In Proposition 1 we have shown that, in every subgame perfect equilibrium of the sequential best-of- $N$ Blotto game, along the equilibrium path players $A$ and $B$ invest $\bar{a} / N>0$ and $\bar{b} / N>0$ into each battle, respectively. Thus, player $A$ wins each individual battle with probability $\rho_{N}=p(\bar{a} / N, \bar{b} / N)$. Using Lemma 4, player $A$ 's probability of winning the tournament can be written as

$$
\pi(\bar{a}, \bar{b}, N)=\sum_{k=n}^{N}\left(\begin{array}{l}
N \\
k
\end{array}\right)\left(\rho_{N}\right)^{k}\left(1-\rho_{N}\right)^{N-k} .
$$

To show part (a) of the result, suppose $\bar{p}>1 / 2$ (if $\bar{p}<1 / 2$ the argument is analogous). There exists $\delta>0$ and $N^{*}$ such that $\rho_{N}>1 / 2+\delta$ for all $N>N^{*}$. Since, for given $N,(23)$ increases in $\rho_{N}$, a lower bound for $\pi(\bar{a}, \bar{b}, N)$ can be obtained by substituting $\rho_{N}=1 / 2+\delta$ into $(23)$ :

$$
\underline{\pi}=\sum_{k=n}^{N}\left(\begin{array}{l}
N \\
k
\end{array}\right)(1 / 2+\delta)^{k}(1 / 2-\delta)^{N-k},
$$

so that $\pi(\bar{a}, \bar{b}, N) \geq \underline{\pi}$ for all $N>N^{*}$. Since $\delta>0$, the Condorcet Jury Theorem (see, e.g., Boland 1998) now implies that $\underline{\pi} \rightarrow 1$, and therefore $\pi(\bar{a}, \bar{b}, N) \rightarrow 1$, as $N \rightarrow \infty$.

To show parts (b) and (c) of the result, suppose $p$ is homogeneous of degree zero and symmetric. Then

$$
\rho_{N}=p(\bar{a} / N, \bar{b} / N)=p(\bar{a}, \bar{b}) \gtreqless 1 / 2 \quad \text { iff } \quad \bar{a} \gtreqless \bar{b}
$$

for all $N$. Thus, the Condorcet Jury Theorem can be applied directly to show that $\bar{a}>\bar{b}$ implies $\pi(\bar{a}, \bar{b}, N)$ is strictly increasing in $N$, and $\pi(\bar{a}, \bar{b}, N) \rightarrow 1$ as $N \rightarrow \infty$. (The results for $\bar{a}<\bar{b}$ and $\bar{a}=\bar{b}$ are analogous.)

\section{Proof of Proposition 5}

As we explained in the text, in anticipation of the even-split equilibrium at the second stage of the extended model, we can view the fundraising stage as a one-shot contest in which $A$ and $B$ invest efforts $x$ and $y$, pay linear costs $C(x)=x$ and $C(y)=y$, and $A$ wins with probability

$$
\pi(x, y) \equiv \sum_{k=n}^{N}\left(\begin{array}{l}
N \\
k
\end{array}\right) p(x, y)^{k}(1-p(x, y))^{N-k},
$$


where $p$ is a symmetric and h.o.d. 0 and $N>0$ is odd. Efforts larger than one are not individually rational; hence the strategy space is $[0,1] \times[0,1]$. Note that the properties of $p$ (continuity on $\mathbb{R}_{+}^{2} \backslash(0,0)$, symmetry, monotonicity in $x$ and $y$, homogeneity) transfer to $\pi$.

Let $\bar{\pi}=\pi(x, 0)$ (any $x>0$ ) and $\underline{\pi}=\pi(0, y)$ (any $y>0$ ), and for $\lambda \in(0, \infty)$ define

$$
q(\lambda) \equiv \pi(x, \lambda x) \quad(\text { where } x>0 \text { is arbitrary) }
$$

This means: $q(\lambda)$ is player $A$ 's success probability in the contest if $B$ spends $\lambda$ times the amount spent by $A$. Continuity of $\pi$ implies $\underline{\pi}<q(\lambda)<\bar{\pi}$ for all $\lambda \in(0, \infty)$; monotonicity implies $q^{\prime}(\lambda)<0$; symmetry implies $\underline{\pi}=1-\bar{\pi}<1 / 2$ and $q(\lambda)=1-q(1 / \lambda)$.

For $\varepsilon \in(\underline{\pi}, 1 / 2)$, let $\lambda(\varepsilon)>1$ be the unique $\lambda$ that solves

$$
q(\lambda)=\varepsilon \quad(\text { equivalently: } q(1 / \lambda)=1-\varepsilon) .
$$

This means: In order to hold $A$ to a success probability of $\varepsilon, B$ must spend $\lambda(\varepsilon)$ times the amount $A$ spends. Equivalently, $A$ wins with probability $1-\varepsilon$ if $B$ spends $1 / \lambda(\varepsilon)$ times the amount $A$ spends.

Now compare $\pi$ to the all-pay CSF $p^{\text {all-pay }}$ in (16). Since we restrict attention to $(x, y) \in[0,1] \times[0,1]$, we have

$$
\underline{p} \equiv\left|\pi(x, y)-p^{\text {all-pay }}(x, y)\right| \geq \varepsilon \quad \text { iff } y \in\left[\frac{1}{\lambda(\varepsilon)} x, \min \{1, \lambda(\varepsilon) x\}\right] .
$$

The length of the interval $[x / \lambda(\varepsilon), \min \{1, \lambda(\varepsilon) x\}]$ is at most $1-1 / \lambda(\varepsilon)^{2}$. Proposition 3 implies that $\pi(x, y) \rightarrow p^{\text {all-pay }}(x, y)$ pointwise as $N \rightarrow \infty$. Therefore, $\underline{p} \rightarrow 0$ and (for given $\varepsilon) \lambda(\varepsilon) \rightarrow 1$ and $1-1 / \lambda(\varepsilon)^{2} \rightarrow 0$. Together with (24), this implies the following:

Fix $\varepsilon \in(0,1 / 2)$ and suppose $y \sim U[0,1]$. There exists $N^{*}$ such

$$
N>N^{*} \Rightarrow \forall x \in[0,1]: \operatorname{Pr}[\underline{p}>\varepsilon]<\varepsilon
$$

It now follows that the expected payoff that $A$ obtains when he spends $x \in[0,1]$ against the mixed strategy $y \sim U[0,1]$ satisfies

$$
E u^{A}(x, \cdot)=\int_{0}^{1} \pi(x, y) d y-x \rightarrow \int_{0}^{1} p^{\text {all-pay }}(x, y) d y-x \quad \text { uniformly as } N \rightarrow \infty
$$

But we know that $A$ 's best response to mixed strategy $y \sim[0,1]$ is to play mixed strategy $x \sim U[0,1]$, which means that every $x \in[0,1]$ results in the same expected maximized 
payoff, which is 0 (see Baye et al. 1996). Thus, (25) becomes

$$
E u^{A}(x, \cdot)=\int_{0}^{1} \pi(x, y) d y-x \rightarrow 0 \quad \text { uniformly as } N \rightarrow \infty .
$$

Thus, for any $\varepsilon>0$, if $N$ is sufficiently large $E u^{A}(x, \cdot) \leq \varepsilon$ for any $x \in[0,1]$, and $\left|E u^{A}(x, \cdot)-E u^{A}\left(x^{\prime}, \cdot\right)\right| \leq \varepsilon$ for any $x, x^{\prime} \in[0,1]$. Reversing the roles of the players, the same can be shown for $B$ 's expected payoffs when $x \sim[0,1]$. The result follows.

\section{References}

[1] Aldrich, John H. (1980). "A Dynamic Model of Presidential Nomination Campaigns." American Political Science Review 74 (3), 651-669.

[2] Bagh, Adib (2010). "Variational Convergence: Approximation and Existence of Equilibria in Discontinuous Games." Journal of Economic Theory 145, 1244-1268.

[3] Baye, Michael R., Dan Kovenock, and Casper G. de Vries (1996). "The All-Pay Auction With Complete Information." Economic Theory 8 (2), 291-305.

[4] Boland, Philip J. (1998). "Majority Systems and the Condorcet Jury Theorem." Journal of the Royal Statistical Society Series D (The Statistician) 38 (3), 181-189.

[5] Borel, Émile (1921). "La theorie du jeu les equations integrales a noyau symetrique." Comptes Rendus del Academie 173, 1304-1308. English translation by Savage, L. (1953), "The Theory of Play and Integral Equations With Skew Symmetric Kernels," Econometrica 21, 97-100.

[6] Börgers, Tilman (1991). "Upper Hemicontinuity of the Correspondence of SubgamePerfect Equilibrium Outcomes." Journal of Mathematical Economics 20, 89-106.

[7] Brams, Steven J. and Morton Davis (1982). "Optimal Resource Allocation in Presidential Primaries." Mathematical Social Sciences 3(4), 373-388.

[8] Dasgupta, Partha and Eric Maskin (1986). "The Existence of Equilibrium in Discontinuous Economic Games, II: Applications." Review of Economic Studies 53 (1), $27-41$.

[9] Deck, Cary A. and Roman M. Sheremeta (2012). "Fight or Flight? Defending Against Sequential Attacks in the Game of Siege." Journal of Conflict Resolution 56 (6), 1069-1088.

[10] Ferrall, Christopher and Anthony A. Smith, Jr. (1999). "A Sequential Game Model of Sports Championship Series: Theory and Estimation." Review of Economics and Statistics 81 (4), 704-719. 
[11] Fu, Qiang and Jingfeng Lu (2012). "The Optimal Multi-Stage Contest." Economic Theory 51 (2), 351-382.

[12] Gelder, Alan (2014), "From Custer to Thermopylae: Last Stand Behavior in MultiStage Contests." Games and Economic Behavior 87, 442-466.

[13] Gradstein, Mark and Kai A. Konrad (1999). "Orchestrating Rent Seeking Contests." Economic Journal 109 (458), 536-545.

[14] Harbaugh, Rick and Tilman Klumpp (2005). "Early Round Upsets and Championship Blowouts." Economic Inquiry 43, 316-329.

[15] Harris, Christopher and John Vickers (1987). "Racing With Uncertainty." Review of Economic Studies 54(1), 1-21.

[16] Hillman, Arye L. and John G. Riley (1989). "Politically Contestable Rents and Transfers." Economics \& Politics 1(1), 17-39.

[17] Hummel, Patrick and Brian Knight (2015). "Sequential or Simultaneous Elections? A Welfare Analysis." International Economic Review 56 (3), 851-887.

[18] Klumpp, Tilman, Hugo M. Mialon, and Michael A. Williams (2015). "Leveling the Playing Field? The Role of Public Campaign Funding in Elections." American Law and Economics Review 17, 361-408.

[19] Klumpp, Tilman and Mattias K. Polborn (2006). "Primaries and the New Hampshire Effect." Journal of Public Economics 90, 1073-1114.

[20] Konrad, Kai A. (2009). Strategy and Dynamics in Contests. Oxford University Press.

[21] Konrad, Kai A. (2017). "Budget and Effort Choice in Sequential Colonel Blotto Campaigns." Unpublished manuscript.

[22] Konrad, Kai A. and Dan Kovenock (2009). "Multi-Battle Contests." Games and Economic Behavior 66, 256-274.

[23] Kovenock, Dan and Brian Roberson (2010). "Conflicts With Multiple Battlefields." CESifo working paper: Empirical and Theoretical Methods, No. 3165.

[24] Kvasov, Dmitriy (2007). "Contests With Limited Resources." Journal of Economic Theory 127, 738-748.

[25] Malueg, David A. and Andrew J. Yates (2010). "Testing Contest Theory: Evidence from Best-of-Three Tennis Matches." Review of Economics and Statistics 92 (3), 689-692. 
[26] Rinott, Yosef, Marco Scarsini, and Yaming Yu (2012). "A Colonel Blotto Gladiator Game." Mathematics of Operations Research 37 (4), 574-590.

[27] Roberson, Brian (2006). "The Colonel Blotto Game." Economic Theory 29, 1-24.

[28] Roberson, Brian and Dmitriy Kvasov (2012). "The Non-Constant-Sum Colonel Blotto Game." Economic Theory 51, 397-433.

[29] Rosen, Sherwin (1986). "Prizes and Incentives in Elimination Tournaments." American Economic Review 76 (4), 701-715.

[30] Sela, Aner and Eyal Erez (2013). "Dynamic Contests With Resource Constraints." Social Choice and Welfare 41 (4), 863-882.

[31] Taylor, Andrew J. (2010). "Does Presidential Primary and Caucus Order Affect Policy?" Political Research Quarterly 63 (2), 398-409.

[32] Tullock, Gordon (1980). "Efficient Rent-Seeking." In: J. M. Buchanan, R. D. Tollison, and G. Tullock (eds.) Toward a Theory of the Rent-Seeking Society. College Station, Texas. 